\title{
Analysis of Etiology and Clinical Characteristics of 1170 Patients with Symptomatic Epilepsy in Jianghan Plain
}

\author{
Yunyu Ding, Xianglin Cheng* \\ Department of Neurology, The First Clinical College of Yangtze University, Jinzhou, China \\ Email: 445152951@qq.com, *45423626@qq.com
}

How to cite this paper: Ding, Y.Y. and Cheng, X.L. (2020) Analysis of Etiology and Clinical Characteristics of 1170 Patients with Symptomatic Epilepsy in Jianghan Plain. Yangtze Medicine, 4, 132-139. https://doi.org/10.4236/ym.2020.42013

Received: June 16, 2019

Accepted: June 26, 2020

Published: June 29, 2020

Copyright (๑) 2020 by author(s) and Scientific Research Publishing Inc. This work is licensed under the Creative Commons Attribution International License (CC BY 4.0).

http://creativecommons.org/licenses/by/4.0/

\section{Open Access}

\begin{abstract}
Objective: The etiology, clinical manifestations and treatment of 1170 patients with symptomatic epilepsy were retrospectively analyzed and provided guidance for further treatment of symptomatic epilepsy. Method: A retrospective analysis of 1170 cases of symptomatic epilepsy admitted to the First Affiliated Hospital of Yangtze University from January 2013 to June 2018 was conducted to analyze the etiology, clinical manifestations and treatment of different age groups. Results: Symptomatic epilepsy is high in the elderly, and men are more than women. Among the 1170 patients with symptomatic epilepsy, there were $81(6.9 \%)$ cases of brain injury, 721 (61.6\%) cases of cerebrovascular disease, $223(19.0 \%)$ cases of tumor, 59 (5.0\%) cases of central nervous system infection, $33(2.8 \%)$ cases of immune metabolic disease, 11 $(0.9 \%)$ cases of drugs, $6(0.5 \%)$ cases of cortical dysplasia, $6(0.5 \%)$ cases of neurodegenerative disease, $4(0.3 \%)$ cases of parasites, $4(0.3 \%)$ cases of poisons, and $22(1.9 \%)$ cases of other diseases. Among them, central nervous system infection and cerebrovascular disease ranked first among the patients aged 0 - 18 years, followed by immune metabolic diseases; cerebrovascular disease ranked first among the patients aged 19 - 39 years, followed by tumors and brain trauma; cerebrovascular disease ranked first among the patients aged 40 - 65 years, followed by tumors and brain trauma; cerebrovascular disease ranked first among the patients aged over 65 years, followed by tumors, among the types of seizures, 74 (6.3\%) cases of tonic seizures, 68 (5.8\%) cases of clonic seizures, $856(73.2 \%)$ cases of tonic-clonic seizures, 27 (2.3\%) cases of myoclonic seizures, $12(1.0 \%)$ cases of absence seizures, $116(9.9 \%)$ cases of complicated partial seizures, and 17 (1.5\%) cases of partial seizures, in addition to the etiology, $795(67.9 \%)$ cases of sodium valproate, 152 (13.0\%) cases of carbamazepine, 56 (4.8\%) cases of topiramate, $117(10.0 \%)$ cases of levetiracetam, $23(2.0 \%)$ cases of lamotrigine, and 27 (2.3\%) cases of
\end{abstract}


others were taken. Conclusion: In symptomatic epilepsy, different age groups have different causes. Among them, cerebrovascular disease, central nervous system infection, brain trauma, and brain tumor are the main causes. The most common type of seizures is the occurrence of tonic-clonic seizures. The most common drugs are sodium valproate and carbamazepine.

\section{Keywords}

Symptomatic Epilepsy, Etiology, Clinical Features, Treatment

\section{Introduction}

Epilepsy [1] is a clinical syndrome caused by high synchronization abnormal discharges of neurons in the brain caused by various reasons. Its clinical manifestations are paroxysmal, transient, repetitive and stereotyped. A group of epilepsy syndrome is composed of similar symptoms and signs. It is currently believed that epilepsy includes idiopathic epilepsy, symptomatic epilepsy, and cryptogenic epilepsy. Among them, symptomatic epilepsy is the most common, also known as secondary epilepsy, acquired epilepsy, refers to central nervous system lesions or abnormalities due to various reasons (including brain structural abnormalities or various factors affecting brain function). Seizures is one of the symptoms or major symptoms [2]. The etiology of symptomatic epilepsy is very complex, and for patients of different age groups, the etiological distribution is quite different [3]. In infants and young children, the main causes were craniocerebral injury in neonates and medication history in pregnant women. Inbred marriage was the main etiology of epilepsy in rural areas of Ningxia and Lhasa [4] [5], and meningitis or encephalitis was the main etiology of epilepsy in Tiantai county of Zhejiang [6]. The main cause of epilepsy in Dongning county, Heilongjiang province is an infection of the central nervous system [7]. Due to regional differences, the etiology of symptomatic epilepsy is also different in each place. At present, there is no relevant research on the etiology and clinical characteristics of secondary epilepsy in Jianghan plain. Therefore, we retrospectively analyzed the etiology, clinical characteristics and therapeutic drugs of 1170 cases of symptomatic epilepsy in Jianghan Plain to provide guidance for further treatment of symptomatic epilepsy.

\section{Information and Methods}

\subsection{Case Selection}

A retrospective analysis of 1170 cases of symptomatic epilepsy admitted to the First Affiliated Hospital of Yangtze University from January 2013 to June 2018. The gender, age, past history, personal history, family history, EEG monitoring records, neuroimaging data, cerebrospinal fluid results and initial symptoms, and drug treatment were collected. The diagnostic criteria were compiled by the 
Chinese Medical Association as the standard for clinical diagnosis and treatment [8]. Exclude febrile convulsions, migraines, transient ischemic attacks, syncope, pseudoseizures, and unexplained seizures. All patients were given medication to control the onset of epilepsy except for the cause of the disease. This study was approved by the ethics committee of the first clinical affiliated hospital of Changjiang University.

\subsection{Cause Grouping}

According to JiaJianping's seventh edition of neurology [9], the etiology of epilepsy is divided into symptomatic epilepsy, idiopathic epilepsy, and cryptogenic epilepsy. Symptomatic epilepsy is classified according to brain trauma, cerebrovascular disease, brain tumors, central nervous system infections, parasites, metabolic diseases, cortical developmental disorders, neurodegenerative diseases, drugs, poisons, and other definite causes.

1.3 Observe etiological distribution of epilepsy patients of different ages. According to the age of onset, 1170 patients with symptomatic epilepsy were divided into four groups, namely, group 0 - 18 years old, group 19 - 39 years old, group 40 - 65 years old, and group > 65 years old. The components of epilepsy in different age groups were analyzed.

\subsection{Observation Indicators}

Record the number of cases of symptomatic epilepsy patients of different ages, genders and various causes. The number of cases of various seizure types and the number of cases of taking different drugs.

\subsection{Statistical Processing}

The count data was analyzed by chi-square test. The difference was statistically significant at $\mathrm{p}<0.05$.

\section{Results}

\subsection{Age of Onset and Gender Distribution}

Among the 1170 patients with symptomatic epilepsy, 774 (66.2\%) were male and $396(33.8 \%)$ were female; $48(4.1 \%)$ were 0 - 18 years old, $29(2.5 \%)$ were male and $19(1.6 \%)$ were female; $160(13.7 \%)$ were 19 - 39 years old, 101 (8.6\%) were male and $59(5.1 \%)$ were female; There were $612(52.3 \%)$ cases at 40 - 65 years old, 408 (34.9\%) males and 204 (17.4\%) females; 350 (29.9\%) patients over 65 years old, $236(20.2 \%)$ males and 114 (9.7\%) females. Patients over 40 years of age were the high-risk group of symptomatic epilepsy, and male patients were higher than females at all ages, with significant differences $(\mathrm{p}<0.05)$; see Table 1.

\subsection{Etiology Distribution}

1170 cases of symptomatic epilepsy, 81 (6.9\%) cases of brain trauma, 721 
Table 1. Age and sex distribution of 1170 patients with symptomatic epilepsy (cases, \%).

\begin{tabular}{cccc}
\hline Age group (years old) & Male & Female & Total \\
\hline $0-18$ & $29(2.5 \%)$ & $19(1.6 \%)$ & $48(4.1 \%)$ \\
$19-39$ & $101(8.6 \%)$ & $59(5.1 \%)$ & $160(13.7 \%)$ \\
$40-65$ & $408(34.9 \%)$ & $204(17.4 \%)$ & $612(52.3 \%)$ \\
$>65$ & $236(20.2 \%)$ & $114(9.7 \%)$ & $350(29.9 \%)$ \\
Total & $774(66.2 \%)$ & $396(33.8 \%)$ & $1170(100 \%)$ \\
\hline
\end{tabular}

Comment: $\mathrm{p}<0.05$.

(61.6\%) cases of cerebrovascular disease, 223 (19.0\%) cases of tumor, 59 (5.0\%) cases of central nervous system infection, $33(2.8 \%)$ cases of metabolic diseases, $11(0.9 \%)$ cases of drugs, $6(0.5 \%)$ cases of cortical developmental disorders, 6 $(0.5 \%)$ cases of degenerative diseases, $4(0.3 \%)$ cases of parasites, $4(0.3 \%)$ cases of poisons and $22(1.9 \%)$ cases of other diseases. Among them, 0 - 18 years old with central nervous system infection, cerebrovascular disease ranked first, followed by metabolic diseases. At the age of 19 - 39, cerebrovascular disease ranked first, followed by tumors and brain trauma. 40 - 65 years old with cerebrovascular disease ranked first, followed by tumors, brain trauma and so on. Cerebrovascular disease was the first in patients over the age of 65 , followed by tumors. See Table 2.

\subsection{Distribution of Seizure Types}

1170 patients with symptomatic epilepsy, 74 (6.3\%) cases of tonic seizures, 68 (5.8\%) cases of clonic seizures, $856(73.2 \%)$ cases of tonic-clonic seizures, 27 (2.3\%) cases of myoclonic seizures, $12(1.06 \%)$ cases of absence seizures, 116 (9.9\%) cases of complicated partial seizures, Partial seizures 17 (1.5\%) cases. See Table 3.

\subsection{Drug Distribution}

Among the 1170 patients with symptomatic epilepsy, in addition to etiological treatment, there were 795 (67.9\%) patients taking valproate sodium, $152(13.0 \%)$ patients taking carbamazepine, $56(4.8 \%)$ patients taking topiramate, 117 (10.0\%) patients taking levoethiracetam, 23 (2.0\%) patients taking lamotrigine, and $27(2.3 \%)$ patients taking other drugs. See Table 4.

\section{Discussion}

Epilepsy is a common neurological disease that seriously endangers human health. According to recent studies in China, the prevalence of epilepsy in China is $0.9 \%-4.8 \%$, which is at a low level in developing countries [10]. In recent years, with neuroimaging with the development of learning and electrophysiological examination techniques, the etiology of a considerable number of patients with epilepsy has gradually become clear, and more and more patients are 
Table 2. Distribution of etiology of 1170 patients with symptomatic epilepsy (cases, \%).

\begin{tabular}{ccccccccccccc}
\hline $\begin{array}{c}\text { Age } \\
\text { group }\end{array}$ & Trauma & Stroke & Tumor & Infection & Parasite & $\begin{array}{c}\text { Metabolic } \\
\text { cortical }\end{array}$ & $\begin{array}{c}\text { Developmental } \\
\text { disorders }\end{array}$ & $\begin{array}{c}\text { Degenerative } \\
\text { diseases }\end{array}$ & Drug & Poison & Other & Total \\
\hline $0-18$ & 4 & 12 & 2 & 12 & 0 & 10 & 3 & 1 & 0 & 0 & 4 & 48 \\
& $(0.34 \%)$ & $(1.02 \%)$ & $(0.17 \%)$ & $(1.02 \%)$ & $(0.00 \%)$ & $(0.85 \%)$ & $(0.27 \%)$ & $(0.09 \%)$ & $(0.00 \%)$ & $(0.00 \%)$ & $(0.34 \%)$ & $(4.1 \%)$ \\
& 20 & 61 & 44 & 13 & 0 & 6 & 3 & 1 & 9 & 0 & 3 & 160 \\
$19-39$ & $(1.71 \%)$ & $(5.21 \%)$ & $(3.76 \%)$ & $(1.11 \%)$ & $(0.00 \%)$ & $(0.51 \%)$ & $(0.27 \%)$ & $(0.09 \%)$ & $(0.77 \%)$ & $(0.00 \%)$ & $(0.27 \%)$ & $(13.7 \%)$ \\
& 45 & 374 & 131 & 27 & 4 & 14 & 0 & 0 & 2 & 3 & 12 & 612 \\
$40-65$ & $(3.85 \%)$ & $(31.97 \%)$ & $(11.20 \%)$ & $(2.31 \%)$ & $(0.34 \%)$ & $(1.20 \%)$ & $(0.00 \%)$ & $(0.00 \%)$ & $(0.17 \%)$ & $(0.27 \%)$ & $(1.02 \%)$ & $(52.3 \%)$ \\
& 12 & 274 & 46 & 7 & 0 & 3 & 0 & 4 & 0 & 1 & 3 & 350 \\
$>65$ & $(1.02 \%)$ & $(23.42 \%)$ & $(3.93 \%)$ & $(0.60 \%)$ & $(0.00 \%)$ & $(0.27 \%)$ & $(0.00 \%)$ & $(0.34 \%)$ & $(0.00 \%)$ & $(0.09 \%)$ & $(0.27 \%)$ & $(29.9 \%)$ \\
& 81 & 720 & 223 & 59 & 4 & 33 & 6 & 6 & 1 & 4 & 22 & 1170 \\
Total & $(6.9 \%)$ & $(61.6 \%)$ & $(19.0 \%)$ & $(5.0 \%)$ & $(0.3 \%)$ & $(2.8 \%)$ & $(0.5 \%)$ & $(0.5 \%)$ & $(0.9 \%)$ & $(0.4 \%)$ & $(1.90 \%)$ & $(100 \%)$ \\
\hline
\end{tabular}

Table 3. Distribution of seizure types in 1170 patients with symptomatic epilepsy (cases, \%).

\begin{tabular}{ccccccccc}
\hline Age group & Tonic & Clonic & Tonic-clonic & Myoclonic & Absence & Complicated partial & Partial & Total \\
\hline $0-18$ & $7(0.60 \%)$ & $4(0.34 \%)$ & $18(1.53 \%)$ & $2(0.17 \%)$ & $4(0.34 \%)$ & $11(0.94 \%)$ & $2(0.17 \%)$ & $48(4.1 \%)$ \\
$19-39$ & $15(1.30 \%)$ & $7(0.60 \%)$ & $105(9.00 \%)$ & $6(0.50 \%)$ & $2(0.17 \%)$ & $21(1.80 \%)$ & $4(0.34 \%)$ & $160(13.7 \%)$ \\
$40-65$ & $21(1.80 \%)$ & $18(1.53 \%)$ & $505(43.17 \%)$ & $7(0.60 \%)$ & $3(0.26 \%)$ & $52(4.44 \%)$ & $6(0.50 \%)$ & $612(52.3 \%)$ \\
$>65$ & $31(2.65 \%)$ & $39(3.33 \%)$ & $228(19.49 \%)$ & $12(1.06 \%)$ & $3(0.26 \%)$ & $32(2.73 \%)$ & $5(0.43 \%)$ & $350(29.9 \%)$ \\
Total & $74(6.3 \%)$ & $68(5.8 \%)$ & $856(73.2 \%)$ & $27(2.3 \%)$ & $12(1.06 \%)$ & $116(9.9 \%)$ & $17(1.5 \%)$ & $1170(100 \%)$ \\
\hline
\end{tabular}

Table 4. Distribution of medications in 1170 patients with symptomatic epilepsy (cases, \%).

\begin{tabular}{cccccccc}
\hline Age group & Valproate & Carbamazepine & Topiramat & Levoethiracetam & Lamotrigine & Other & Total \\
\hline $0-18$ & $28(2.40 \%)$ & $6(0.50 \%)$ & $2(0.17 \%)$ & $5(0.43 \%)$ & $5(0.43 \%)$ & $2(0.17 \%)$ & $48(4.1 \%)$ \\
$19-39$ & $45(3.84 \%)$ & $35(3.00 \%)$ & $19(1.62 \%)$ & $48(4.10 \%)$ & $9(0.77 \%)$ & $4(0.34 \%)$ & $160(13.7 \%)$ \\
$40-65$ & $474(40.51 \%)$ & $58(5.00 \%)$ & $23(1.97 \%)$ & $37(3.16 \%)$ & $8(0.68 \%)$ & $12(1.03 \%)$ & $612(52.3 \%)$ \\
$>65$ & $248(21.20 \%)$ & $53(4.52 \%)$ & $12(1.03 \%)$ & $27(2.31 \%)$ & $1(0.09 \%)$ & $9(0.77 \%)$ & $350(29.9 \%)$ \\
Total & $795(67.9 \%)$ & $152(13.0 \%)$ & $56(4.8 \%)$ & $117(10.0 \%)$ & $23(2.0 \%)$ & $27(2.3 \%)$ & $1170(100 \%)$ \\
\hline
\end{tabular}

diagnosed with symptomatic epilepsy. The etiology of symptomatic epilepsy is complex and diverse. The etiology of patients in different regions and different age groups is quite different, and the clinical manifestations and treatment measures are also different. Therefore, the causes and manifestations of symptomatic epilepsy at different ages in this region are clarified. And treatment measures have important guiding significance for the diagnosis and treatment of such diseases in the future.

We retrospectively analyzed 1170 cases of hospitalized symptomatic epilepsy admitted to our hospital from January 2013 to June 2018. It was found that patients with symptomatic epilepsy had higher incidence of middle-aged and older men, and more men than women. Cerebrovascular disease, brain tumor, central nervous system infection, and brain trauma accounted for the four most com- 
mon causes. This may be related to the fact that our hospital is the largest comprehensive third-class hospital in Jianghan Plain, and there are more elderly and severe patients in our hospital. However, younger patients tend to get more attention and attention and choose higher-level hospital treatment, which may also be one of the factors leading to fewer people under 18 years of age. At the same time, the etiology of different age groups is also different. Central nervous system infections and cerebrovascular diseases rank first among the patients aged 0 - 18 years, followed by metabolic diseases; cerebrovascular diseases rank first among the patients aged $19-39$ years, followed by tumors and brain trauma; cerebrovascular diseases rank first among the patients aged 40 - 65 years, followed by tumors and brain trauma; cerebrovascular diseases rank first among the patients aged over 65 years, followed by tumors, etc. Among all the causes, epilepsy caused by cerebrovascular disease is the most common, especially in middle-aged and elderly patients. The age is the key cause [11], and the proportion of cardiovascular and cerebrovascular accidents increases with age. In this study, 962 (82\%) patients aged 40 years or older had the onset of epilepsy, suggesting that middle and old age is a peak of onset in symptomatic epilepsy patients, and men have more opportunities than women to have epilepsy risk factors. This is consistent with the characteristics of symptomatic epilepsy patients around the world [12] [13], which also briefly confirms the validity of the data in this study. This also suggests that cerebral apoplexy, as one of the diseases with high incidence, mortality and disability rate in China, has far-reaching harm to various regions including Jianghan plain. The screening of risk factors of cerebrovascular disease and the effective implementation of primary and secondary prevention are of great significance [14]. The etiology of brain tumors also increased with age, but this study found that after 65 years of age, the number of epilepsy caused by brain tumors gradually decreased. Considering the possible reason is that the hospital mainly referred to the lower-level hospital for referral, and some patients with brain tumors gradually lost the opportunity to diagnose and treat the disease, and did not go to the higher-level hospital for further treatment.

The classification of seizures is divided into partial (local) seizures, generalized seizures, and unclassified seizures, while generalized seizures are divided into tonic seizures, clonic seizures, tonic-clonic seizures, myoclonic seizures, absence seizures, and No tension episodes. Studies have shown that partial seizures account for $64 \%$ of seizures, while systemic seizures only account for $30 \%$ [15], and some studies have previously shown that seizures are predominantly generalized seizures, accounting for $71.10 \%$ [16]. In this group of cases, there were 74 cases of tonic seizures, 68 cases of clonic seizures, 856 cases of tonic-clonic seizures, 27 cases of myoclonic seizures, 12 cases of absence seizures, 116 cases of complicated partial seizures, and 17 cases of partial seizures. The main inconsistencies with the reports are that Jianghan Plain is located in the central and western part of China and is a traditional agricultural area. Its economic and sanitary conditions are poor. The patients have insufficient knowledge of epilepsy 
seizures. Most patients with partial seizures do not have a diagnosis.

In terms of treatment, the first is etiological treatment, while avoiding predisposing factors such as fatigue, mood swings, lack of sleep, etc., but all patients basically need to choose medication maintenance treatment, In the choice of drugs, single-drug and low-dose treatments were used. All patients took the drug for life. In this group, 795 cases of sodium valproate, 152 cases of carbamazepine, 56 cases of topiramate, 117 cases of levetiracetam, 23 cases of lamotrigine, other 27 cases, Among them, carbamazepine and sodium valproate are the most widely used because carbamazepine is the preferred drug for partial seizures and symptomatic epilepsy in our province. As a broad-spectrum antiepileptic drug, valproic acid has a good effect on all kinds of comprehensive seizures, and the traditional antiepileptic drugs are relatively cheap, which also reduces the economic burden of patients. However, the application of carbamazepine and sodium valproate is further limited due to their skin rash, liver function damage, hematopoietic system damage and teratogenicity. Therefore, in our case, due to their positive clinical effects, minor side effects, and the improvement of people's living standards, the requirements for the safety and side effects of the drugs are higher, leading to their increasing use. However, the low use of to piramate and other drugs may be related to doctors' and patients' insufficient understanding of other new antiepileptic drugs, which needs to be further improved in future clinical work.

After controlling symptoms, most patients with secondary epilepsy often choose to follow-up in nearby hospitals or other general hospitals. It is difficult to track patients' condition, drug use and prognosis. At the same time, this study has limitations. Secondary epilepsy patients can also choose other hospitals for treatment. The data collected by our hospital is limited. We will further cooperate with large-scale general hospitals at county and city levels in Jianghan Plain to share data of epilepsy patients and make the analysis more comprehensive. In short, clear symptomatic epilepsy, seizure form, correct, reasonable choice of antiepileptic drugs, can achieve better clinical treatment effect, further reduce the side effects of drugs, improve the quality of life of patients.

\section{Conflicts of Interest}

The authors declare no conflicts of interest regarding the publication of this paper.

\section{References}

[1] Qiu, W.J., Hu, X.W. and Zhang, Z.C. (2014) Research Progress in the Pathogenesis and Treatment of Epilepsy. Chinese Journal of Clinicians, 10, 1920-1924.

[2] Guo, M.H. and Zhang, J.J. (2013) Investigation and Epidemiological Investigation of Epilepsy. Chinese Journal of Brain Disease and Rehabilitation (Electronic Edition), 3, 338-340.

[3] Wu, J., Chang, L.Y. and Xu, L. (2018) Etiology Analysis of 194 Adult Patients with Symptomatic Epilepsy. Stroke and Nervous Diseases, 25, 316-317. 
[4] China Anti-Epilepsy Association (2015) Clinical Diagnosis and Treatment Guidelines for Epilepsy (2015 Revised Edition). People’s Medical Publishing House, Beijing.

[5] Chen, G.Y., Dai, X.Y., Kong, F.Y., et al. (2003) WHO/IEM/ILAE Global Antiepileptic Campaign-Epidemiological Survey of Epilepsy in Rural Ningxia. Chinese Journal of Neurology, 2, 421.

[6] Da, G.W. (1997) Epidemiological Investigation on Epilepsy of 30,000 Tibetan Peasants in Lhasa. Journal of Tibetan Medicine, 18, 23.

[7] Hong, Z., Wang, X.Y., Huang, M.S., et al. (2000) Epidemiological Investigation of Epilepsy in Rural Areas of Tiantai County, Zhejiang Province. Chinese Journal of Epidemiology, 21, 127.

[8] Wang, D.S., Li, G.Z., Ma, G.Y., et al. (2002) Epidemiological Survey of Epilepsy in Dongning County, Heilongjiang Province, Rural China. Journal of Stroke and Neurological Diseases, 19, 159.

[9] Jia, J.P. and Chen, S.D. (2015) The Seventh Edition of Neurology. People's Medical Publishing House, Beijing, 297.

[10] Wang, W.Z., Wu, J.Z., Wang, D.S., et al. (2003) The Prevalence and Treatment Gapin Epilepsy in China: An ILAE/IBE/WHO Study. Neurology, 60, 1544-1545. https://doi.org/10.1212/01.WNL.0000059867.35547.DE

[11] Zuo, Q.H. (2005) Pediatric Nervous System Diseases. People's Medical Publishing House, Beijing, 216.

[12] Tian, H.J., Wang, X.Q., Shi, X.B., et al. (2015) Evaluation of Clinical Features of Epilepsy in China. International Journal of Clinical and Experimental Medicine, 8, 2399-2404.

[13] Guekht, A., Hauser, W.A., Milchakova, L., et al. (2010) The Epilemiology of Epilepsy in the Russian Federation. Epilepsy Research, 92, 209-218. https://doi.org/10.1016/j.eplepsyres.2010.09.011

[14] Cerebrovascular Group, Neurology Branch, Chinese Medical Association (2015) Chinese Guidelines for First-Level Prevention of Cerebrovascular Diseases 2015. Chinese Medical Information, 30, 8.

[15] Syvertsen, M., Nakken, K.O., Edland, A., et al. (2015) Prevalence and Etiology of Epilepsy in a Norwegian County-A Population Based Study. Epilepsia, 56, 699-706. https://doi.org/10.1111/epi.12972

[16] Fawi, G., Khedr, E.M., Elfetoh, N.A., et al. (2015) Community-Based Epidemiological Study of Epilepsy in the Qena Governorate in Upper Egypt, a Door-to-Door Survey. Epilepsy Research, 113, 68-75.

https://doi.org/10.1016/j.eplepsyres.2015.03.010 